\title{
Dirac Quantization of Open Strings and Noncommutativity in Branes
}

\author{
F. Ardalan ${ }^{a, b}$, H. Arfaei ${ }^{a, b}$ and M.M. Sheikh-Jabbari ${ }^{a}$ \& \\ a Institute for Studies in Theoretical Physics and Mathematics IPM, \\ P.O.Box 19395-5531, Tehran, Iran \\ and \\ ${ }^{b}$ Department of Physics Sharif University of Technology, \\ P.O.Box 11365-9161, Tehran, Iran
}

\begin{abstract}
We apply the Dirac bracket quantization to open strings attached to branes in the presence of background antisymmetric field and recover an inherent noncommutativity in the internal coordinates of the brane.
\end{abstract}

${ }^{1}$ E-mail: ardalan, arfaei, jabbari@theory.ipm.ac.ir 


\section{Introduction}

Since the discovery of the role of branes in string theory [1] they have frequently shown unexpected properties. They were first identified as the carriers of R-R charges and very soon after, it was realized that when $N$ of them merge the space-time coordinates normal to them become noncommutative [2] and the $U(N)$ Super-Yang-Mills theory emerges. Another type of noncommutativity appears in the bound states of branes with fundamental strings and with other branes ft. It has been shown that such brane bound states correspond to branes with non-zero background internal gauge fields $[2,3,4]$. These are the type of branes we shall be focusing on in this article. The correspondence has been demonstrated by constructing the interaction amplitudes of two branes [4,5] and by looking at the scattering of different closed strings off the branes [6]. The noncommutativity arising in the internal structure of these brane bound states is a consequence of the properties of open strings ending on them. Such open strings satisfy boundary conditions which are neither Neumann nor Dirichlet, but a combination of the two, sometimes referred to as mixed boundary condition $[4,5,7]$. The mixed boundary condition makes the canonical quantization of the theory non-trivial. Imposing the standard commutation relations, leads to inconsistency. It has been proposed to remove the inconsistency by relaxing the commutativity of the space coordinates of the open strings along the direction of the brane described by mixed boundary conditions $[7,8,9]$. The procedure of relaxing the commutativity of space coordinates adopted in $[5,8]$, was to keep the standard algebra of the Fourier modes in the mode expansion.

The noncommutativity observed in the above brane system seemed very similar to that observed by Connes, Douglas, Schwartz (CDS) [10] in the problem of Matrix Model with non-trivial background three form. They studied compactification of Matrix theory on a noncommutative torus and realized that it corresponds to the Matrix theory in such backgrounds.

Motivated by this observation, we showed that $[7,8]$, the noncommutativity can be derived within the string theory by wrapping branes with non-zero $B_{i j}$ background field on the compactification torus. Moreover in [8] the $C^{*}$-algebra of functions on noncommutative torus which is building block of the Connes' noncommutative geometry construction, [10,11], was also constructed using the noncommutative open string position operators.

The BPS spectrum of Yang-Mills theory on noncommutative torus was also originally

\footnotetext{
${ }^{2}$ Another type of noncommutativity appeared in the context f $2+1$ dimensional Chern-Simons theory
} $[26]$. 
obtained by CDS. Later the spectrum of the noncommutative M(atrix)-model was derived by taking the longitudinal and transverse membrane winding into account [12]. As another evidence for the above correspondence, in $[8,13]$ it was shown that the low energy BPS spectrum of such brane system is exactly the same as the results of $[10,12]$.

Soon after [10], another check on this correspondence was made by Douglas and Hull [14]. They showed that string compactification, along with wrapping a two-brane in the non-zero two-from background, $B_{i j}$, in the low energy limit leads to Yang-Mills theory on the dual noncommutative torus. Studying the three and four open strings scattering amplitudes in [15], the noncommutative Yang-Mills theory was obtained as the related low energy effective field theory on the branes. Further evidence for the correspondence of the work of CDS and string theory in a non-zero $B_{\mu \nu}$ background field has been discussed in many papers [16]. Despite these checks which support the noncommutativity of the different position coordinates of the brane with non zero background B-field, the arguments leading to noncommutativity of space coordinates was based on a number of assumptions which might be open to question. The method adopted in $[5,8]$ has been based on taking the commutation relation among the raising and lowering operators of the open strings in absence of the $B_{\mu \nu}$ field and imposing the commutativity on the zero modes. On the other hand in [9] the analysis was based a time averaged symplectic form.

In this article we will try to provide a solid ground for the quantization of this system. Since the serious obstacle in the way of quantization is the mixed boundary condition we need to treat it carefully. We consider it as a constraint and apply the method of Dirac to find the commutators. In the rest of the introduction we give a brief review of the related matters, the Matrix model on the noncommutative torus, the previous approaches to the quantization of strings attached to branes with background B fields, and an outline of the Dirac method [17].

In the $\mathrm{M}$ (atrix)-model one considers a set of $N \times N$ bosonic hermitian matrices, $X^{a}$, $a=$ $1, \ldots, 9$ and sixteen component spinor of $S O(9), \Psi$, and dynamics of these matrices is given by

$$
I=\frac{1}{2 g \sqrt{\alpha^{\prime}}} \int d \tau \operatorname{Tr}\left\{\dot{X}_{a} \dot{X}_{a}+\frac{1}{\left(2 \pi \alpha^{\prime}\right)^{2}} \sum_{a<b}\left[X^{a}, X^{b}\right]^{2}+\frac{i}{2 \pi \alpha^{\prime}} \Psi^{T} \dot{\Psi}-\frac{1}{\left(2 \pi \alpha^{\prime}\right)^{2}} \Psi^{T} \Gamma_{a}\left[X^{a}, \Psi\right]\right\} .
$$

M(atrix) model was conjectured to be a light cone formulation of M-theory [18]. Thus, although the theory originally involves eleven dimensions, it has nine dimensional objects as functions of time. The above conjecture has passed many consistency checks [19], one of the most important of them was its compactifications on different manifolds. Compactification 
of the matrix coordinate $X^{a}$ on a circle of radius $R$ is possible if there exist matrices $U$ as a part of the symmetries of (1.1) that shifts $X^{a}$ by $R$;

$$
U X^{a} U^{-1}=X^{a}+R
$$

leaving the other coordinates and spinors unchanged. Modding out the action of $U$ is equivalent to compactification. This $S^{1}$ compactification leads to the string Matrix theory of Dijkgraaf-Verlinde-Verlinde [20] which is shown to be equivalent to type IIA free string theory. Compactification on higher dimensional tori is given similarly;

$$
\begin{gathered}
U_{i} X_{i} U_{i}^{-1}=X_{i}+R_{i}, \\
U_{i} X_{j} U_{i}^{-1}=X_{j} \quad i \neq j, \\
U_{i} \Psi U_{i}^{-1}=\Psi .
\end{gathered}
$$

Consistency requires that $U_{i}$ 's should commute up to a phase;

$$
U_{i} U_{j}=e^{i \theta_{i j}} U_{j} U_{i}
$$

For ordinary commutative torus $U_{i}$ 's are translation operators with $\theta_{i j}=0$ 甲. If $\sigma_{i}$ 's parameterize the dual torus, a solution to (1.3) for $\theta_{i j}=0$ is

$$
\begin{gathered}
X_{i}=i \frac{\partial}{\partial \sigma_{i}}+A_{i}(\sigma), \\
U_{i}=e^{i \sigma_{i} R_{i}},
\end{gathered}
$$

It is easily seen that such compactification yields the Yang-Mills theory on the dual torus.

The major step taken by CDS, [10], was to realize that non-zero, irrational $\theta_{i j}$ leads to compactification on a noncommutative torus, and the solution to (1.3) at the limit of large $N$ describes a Yang-Mills theory on the dual noncommutative torus in which we have to replace the definition of commutators with the Moyal brackets:

$$
\begin{gathered}
\{A, B\}=A * B-B * A \\
A * B(\sigma)=\left.e^{-i \theta\left(\partial_{1}^{\prime} \partial_{2}^{\prime \prime}-\partial_{2}^{\prime} \partial_{1}^{\prime \prime}\right)} A\left(\sigma^{\prime}\right) B\left(\sigma^{\prime \prime}\right)\right|_{\sigma^{\prime}=\sigma^{\prime \prime}=\sigma} .
\end{gathered}
$$

\footnotetext{
${ }^{3}$ Due to SL(2,Z)-type symmetries [11] rational $\theta_{i j}$ 's can be mapped to an integer and hence they also result in commutative space.
} 
Non-zero $\theta_{i j}$ leads to non-locality in the gauge theory and break down of Lorentz invariance. This has led to a line of investigation on Moyal gauge theories [21]. In their original work CDS attempt to identify physical sources of noncommutativity and suggest that it is due to non-zero $C_{-i j}$, the three form as a background, when one formulates the M-theory on the light cone.

We will now review the two previous approaches of mixed open strings quantization. We take the open strings having their ends on non-marginal BPS bound states of $\mathrm{p}, \mathrm{p}-2, \mathrm{p}-4, \ldots$ branes. Such bound states are represented by a non-zero static and uniform configuration of $B_{\mu \nu}$ field in the bulk and $A_{\mu}$ field with constant strength, $F_{\mu \nu}$, in the brane. Suppose the brane spans a $\mathrm{p}+1$ dimensional space $\left(X^{0}, X^{1}, \ldots, X^{p}\right)$ with $X^{p+1}=X^{p+2}=\ldots=X^{9}=0$. Action for the open string with its ends on this brane is [22];

$$
\begin{gathered}
S=\frac{1}{4 \pi \alpha^{\prime}} \int_{\Sigma} d^{2} \sigma\left[\eta_{\mu \nu} \partial_{a} X^{\mu} \partial_{b} X^{\nu} g^{a b}+\epsilon^{a b} B_{\mu \nu} \partial_{a} X^{\mu} \partial_{b} X^{\nu}\right]+ \\
\left.\frac{1}{2 \pi \alpha^{\prime}} \int d \tau A_{i} \partial_{\tau} X^{i}\right|_{\pi}-\left.\frac{1}{2 \pi \alpha^{\prime}} \int d \tau A_{i} \partial_{\tau} X^{i}\right|_{0}
\end{gathered}
$$

where $A_{i}, i=0,1, \ldots, p$ is the $U(1)$ gauge field living on the D-brane. The symmetries of the action includes on top of the geometric symmetries, two $U(1)$ gauge symmetries, one of them, which we call it $\lambda$-gauge symmetry, only acts on the internal field:

$$
A \rightarrow A+d \lambda
$$

and this is the symmetry which raises to $U(N)$ symmetry when $N$ branes coincide. The other $U(1)$ symmetry, which we will note it by $\Lambda$-gauge symmetry, acts on both $B_{\mu \nu}$ and $A_{i}$ :

$$
\begin{gathered}
B_{\mu \nu} \rightarrow B_{\mu \nu}+\partial_{\mu} \Lambda_{\nu}-\partial_{\nu} \Lambda_{\mu} \\
A_{\mu} \rightarrow A_{\mu}+\Lambda_{\mu},
\end{gathered}
$$

and hence the gauge invariant field strength is

$$
\mathcal{F}_{\mu \nu}=B_{\mu \nu}-F_{\mu \nu} \quad, \quad F_{\mu \nu}=d A=\partial_{[\mu} A_{\nu]}
$$

The equations of motion are the same as ordinary open strings but the boundary conditions at ends are neither Neumann nor Dirichlet but a linear combination of the two,

$$
\partial_{\sigma} X^{\mu}+\mathcal{F}_{\mu \nu} \partial_{\tau} X^{\nu}=0 \text { at } \sigma=0, \pi
$$

To quantize the theory we should first find the canonical conjugates of $X^{\mu}$

$$
P_{\mu}(\sigma, \tau)=\frac{\partial S}{\partial X^{\mu}(\sigma, \tau)}=\frac{1}{2 \pi \alpha^{\prime}}\left(\partial_{\tau} X_{\mu}+B_{\mu \nu} \partial_{\sigma} X^{\nu}+A_{\mu}(\delta(\sigma-\pi)-\delta(\sigma)) .\right.
$$


An important point is the freedom in the definition of the conjugate momentum. It depends on the choice of both $U(1)$ gauges discussed above. Therefore it suggests that the noncommutativity may be an artifact of these choices. To clarify this point, in this paper, we do not fix the gauge and conclude that within Dirac's method of quantization although the noncommutativity depends on the gauge, it is not removable by a gauge choice. The physical quantities such as spectrum depend on the gauge invariant field $\mathcal{F}$. The gauge choice of $[4,5]$ and [8] which was also adopted in [9],

$$
A_{\mu}=0 \text { or } \mathcal{F}=B
$$

has no delta function singularity at the ends of the string. In this gauge the conjugate momenta are

$$
2 \pi \alpha^{\prime} P_{\mu}(\sigma, \tau)=\partial_{\tau} X_{\mu}+\mathcal{F}_{\mu \nu} \partial_{\sigma} X^{\nu}
$$

Imposing the canonical quantization conditions proves to be inconsistent and hence we have to search for a remedy. Although in [8] the concentration was on the compact case, the arguments given there hold for the non-compact case too. The method adopted there is to make the commutation relation of the mixed open strings modes satisfy the same algebra as the Neumann open strings.

Taking

$$
\begin{gathered}
{\left[P^{\mu}(\sigma, \tau), P^{\nu}\left(\sigma^{\prime}, \tau\right)\right]=0} \\
{\left[X^{\mu}(\sigma, \tau), P^{\nu}\left(\sigma^{\prime}, \tau\right)\right]=i \eta^{\mu \nu} \delta\left(\sigma-\sigma^{\prime}\right),}
\end{gathered}
$$

as essential commutation relations, and imposing them on the classical mixed open string mode expansions

$$
\begin{gathered}
X^{\mu}=x^{\mu}+p^{\mu} \tau-\mathcal{F}^{\mu \nu} p_{\nu} \sigma+\sum_{n \neq 0} \frac{e^{-i n \tau}}{n}\left(i a_{n}^{\mu} \cos n \sigma-\mathcal{F}_{\nu}^{\mu} a_{n}^{\nu} \sin n \sigma\right) \\
2 \pi \alpha^{\prime} P^{\mu}=\mathcal{M}_{\nu}^{\mu}\left(p^{\mu}+\sum_{n \neq 0} e^{-i n \tau} a_{n}^{\mu} \cos n \sigma\right), \quad \mathcal{M}=\mathbf{1}-\mathcal{F}^{2},
\end{gathered}
$$

we find that

$$
\left[p^{\mu}, p^{\nu}\right]=0 \quad, \quad\left[x^{\mu}, p^{\nu}\right]=2 i \alpha^{\prime}\left(\mathcal{M}^{-1}\right)^{\mu \nu} \quad, \quad\left[a_{n}^{\mu}, a_{m}^{\nu}\right]=2 \alpha^{\prime}\left(\mathcal{M}^{-1}\right)^{\mu \nu} n \delta_{n+m}
$$

Now let us consider the $X^{\mu}, X^{\nu}$ commutators,

$$
\left[X^{\mu}(\sigma, \tau), X^{\nu}\left(\sigma^{\prime}, \tau\right)\right]=\left[x^{\mu}, x^{\nu}\right]+2 i \alpha^{\prime}\left(\mathcal{M}^{-1} \mathcal{F}\right)^{\mu \nu}\left(\sigma+\sigma^{\prime}+\sum_{n \neq 0} \frac{1}{n} \sin n\left(\sigma+\sigma^{\prime}\right)\right) .
$$


The sum appearing in the above equation is

$$
\sigma+\sigma^{\prime}+\sum_{n \neq 0} \frac{1}{n} \sin n\left(\sigma+\sigma^{\prime}\right)=\left\{\begin{array}{cc}
0 & \sigma=\sigma^{\prime}=0 \\
2 \pi & \sigma=\sigma^{\prime}=\pi \\
\pi & \text { otherwise }
\end{array}\right.
$$

and hence

$$
\left[X^{\mu}(\sigma, \tau), X^{\nu}\left(\sigma^{\prime}, \tau\right)\right]=\left\{\begin{array}{cc}
{\left[x^{\mu}, x^{\nu}\right]} & \sigma=\sigma^{\prime}=0 \\
{\left[x^{\mu}, x^{\nu}\right]+4 \pi i \alpha^{\prime}\left(\mathcal{M}^{-1} \mathcal{F}\right)^{\mu \nu}} & \sigma=\sigma^{\prime}=\pi \\
{\left[x^{\mu}, x^{\nu}\right]+2 \pi i \alpha^{\prime}\left(\mathcal{M}^{-1} \mathcal{F}\right)^{\mu \nu}} & \text { otherwise. }
\end{array}\right.
$$

In this intuitive quantization method $\left[x^{\mu}, x^{\nu}\right]$ is not determined and can be chosen arbitrarily. In $[8]\left[x^{\mu}, x^{\nu}\right]$ was chosen to be zero and hence the open strings center of mass became noncommuting. This choice was supported by the following physical argument: To find out whether the space is commuting or not, we should probe it by strings, open or closed. Since the closed strings live in the bulk and not only on the branes, they will not show the noncommutative structure of the space [6]. In a more intuitive explanation since the "right" and "left" moving modes of closed strings contribute equally in the probing processes, the noncommutativity seen by the left movers cancel those of right movers and so the space probed by closed strings is commutative. When we use the mixed open strings as probes because of asymmetry in the "left" and "right" modes, the space seen by such strings is noncommuting. And the related physical quantity which shows the space noncommutativity is the center of mass coordinates.

In another quantization approach, authors of [9] tried to fix the arbitrariness of $\left[x^{\mu}, x^{\nu}\right]$. They proposed to use the "time averaged" symplectic form:

$$
\tilde{\Omega}=\frac{1}{2 \alpha^{\prime}}\left\{\mathcal{M}_{\mu \nu} d p^{\mu}\left(d x^{\nu}+\frac{\pi}{2} \mathcal{F}_{\nu \alpha} d p^{\alpha}\right)\right\}+\sum_{n>0} \frac{-i}{n}\left(\mathcal{M}_{\mu \nu} d a_{n}^{\mu} d_{n}^{\nu}\right) .
$$

This method leads to

$$
\left[x^{\mu}, x^{\nu}\right]=-2 \pi i \alpha^{\prime}\left(\mathcal{M}^{-1} \mathcal{F}\right)^{\mu \nu}
$$


Plugging (1.22) into (1.20), they obtained

$$
\left[X^{\mu}(\sigma, \tau), X^{\nu}\left(\sigma^{\prime}, \tau\right)\right]=\left\{\begin{array}{c}
-2 \pi i \alpha^{\prime}\left(\mathcal{M}^{-1} \mathcal{F}\right)^{\mu \nu}, \text { for } \sigma=\sigma^{\prime}=0 \\
2 \pi i \alpha^{\prime}\left(\mathcal{M}^{-1} \mathcal{F}\right)^{\mu \nu}, \text { for } \sigma=\sigma^{\prime}=\pi \\
0 \quad \text { otherwise. }
\end{array}\right.
$$

In other words, they concluded that mixed open strings are noncommutating only at the ends, where open strings are attached to the brane, i.e. $\sigma=\sigma^{\prime}=0, \sigma=\sigma^{\prime}=\pi$, and the center of mass coordinates are commuting.

To settle the question of the seat of noncommutativity in a more fundamental point of view, since the problem arises from the boundary conditions which can be treated as constraints, we take the Dirac's quantization method to analyze the problem [17], which we will now outline:

Suppose we take a system with the canonical position and momenta $\left(q_{i}, p_{i}\right) ; i=1, \ldots, n$ and assume that they are constrained to lie on the hypersurfaces $\Phi_{m}(q, p)=0, m=1, \ldots, r$. In studying this system we face two problems;

i) The Hamiltonian is not well-defined and can be modified by adding a linear combination of the constraints; and

ii) The dynamics may not respect the constraints.

In the Dirac's method both difficulties are resolved. First, if the constraints are violated under time evolution, by taking the Poisson bracket of Hamiltonian and the constraints, we may construct a larger set of constraints which are consistent with dynamics. Furthermore the freedom in the Hamiltonian may also be fixed by this consistency. The final step to construct the Dirac brackets is to check the consistency of the constraints, i.e. consider the Poisson brackets of the constraints. If they turn out to be zero, the constraints are called first class and they are consequence of symmetries of the system. If it happens that the constraints have non-vanishing Poisson brackets, and the matrix of their brackets is nonsingular, the second class constraints, Dirac defines the quantum commutator in terms of a modified Poisson bracket, i.e. Dirac bracket. It is interesting that in the case of second class constraints we can find a new set of canonical variables in which the constraints are practically decoupled from the physical degrees of freedom [23,24] and the Poisson brackets of the new set of variables are the same as Dirac brackets. We take the boundary conditions as constraints and follow the procedure described above. The constraints are of second class. We find that the coordinates at the ends points of the strings are noncommutative. The 
exact value of the commutators depend on the $\Lambda$-gauge we choose, but as we can see from its expression given below, is non-zero in any gauge f?

$$
\left[X^{i}(0, \tau), X^{j}(0, \tau)\right]=2 \pi \alpha^{\prime} i\left(\left(-\mathcal{M}+\frac{1}{2} F \mathcal{F}\right)^{-1} \mathcal{F}\right)^{i j}
$$

This is unlike the commutators of the momenta which can be set to zero for all string points in the gauge $F=0$. This gauge is particularly useful since the mixed open strings momentum distribution does not possess delta function singularity at the end of the string.

In section 2, we review the method of Dirac quantization in detail. In section 3, we apply it to the well-known cases of D-branes and show the equivalence of the Dirac method and the usual mode expansion and quantizing the Fourier modes. In section 4, applying Dirac's method to the case of non-zero background antisymmetric field, i.e. the case of mixed boundary conditions, we show that despite gauge dependence, the noncommutativity is not removable. In the Dirac's prescription, unlike the mode expansion method, the boundary conditions at two ends of open strings appear independently, so we can consider the strings with their ends on the same or different branes. In this section, we also compare our results with the previous methods, and argue that in a gauge in which the internal $U(1)$ field is set to zero, the results of Dirac quantization agree with the mode expansion method provided we set the commutators of the center of mass coordinates to zero. The last section is devoted to conclusions and discussions.

\section{Review of Dirac Quantization}

According to principles of quantum mechanics, to quantize a mechanical system a Hamiltonian formulation of the system is needed, i.e. a Hamiltonian and a Poisson bracket structure. Having these two we can find the dynamics of any function defined on the phase space of the theory, and to go over to the quantized theory we should replace the Poisson bracket with the commutators. But, in general, when the theory is subject to constraints, i.e. when all of the phase space is not available for the motion, the Hamiltonian formulation should be modified. Dirac has given a concise recipe to handle these constrained systems [17], which we briefly review here.

Given a Hamiltonian, $H$, and a set of primary constraints, $\Phi_{m}(q, p)=0$, since any linear combination of the constraints is also zero, the Hamiltonian is not uniquely determined and

\footnotetext{
${ }^{4}$ we will present the calculations in section 4.
} 
$H_{T}=H+u_{m} \Phi_{m}$ is as good as $H, u_{m}$ being arbitrary functions in phase space. Dynamics of any function in phase space, $g(q, p)$, is determined by the Poisson bracket structure,

$$
\dot{g} \approx\left\{g, H_{T}\right\}_{P . B .}
$$

the symbol $\approx$ called weak equivalence implying that, the Poisson brackets should be worked out and then the constraints imposed, thus

$$
\Phi_{m} \approx 0
$$

Consistent dynamics requires that the constraints remain valid under time evolution:

$$
\dot{\Phi}_{m} \approx\left\{\Phi_{m}, H_{T}\right\}_{P . B .} \approx\left\{\Phi_{m}, H\right\}+u_{n}\left\{\Phi_{m}, \Phi_{n}\right\} \approx 0
$$

There are then three possibilities

i) The equation $(2.3)$ reduces to $0=0$, i.e. identically satisfied with the imposition of the primary constraints.

ii) They reduce to equations independent of $u$ 's, thus involving only q's and p's. Such equations must be independent of the primary constraints, otherwise they would be of the first kind, thus they are a new set of constraints:

$$
\Psi_{i}(q, p)=0
$$

iii) The equations (2.3) may not reduce in either of these ways; they then may be solved in terms of the $u$ 's.

Equations obtained from the kind ii), (2.4), are called secondary constraints. If a secondary constraint turns up, then their consistency with the time evolution should still be worked out:

$$
\dot{\Psi}_{i} \approx\left\{\Psi_{i}, H_{T}\right\}_{P . B .} \approx 0
$$

These equations should be treated on the same footing as (2.3), and the analysis should be repeated. The procedure should be pursued until there are no more secondary constraints and we have exhausted all the consistency conditions. Finally we will be left with a number of secondary constraints and a number of conditions on $u$ 's. The secondary constraints, for many purposes should be treated on the same footing as the primary ones; e.g. , in evaluating the weak equations they should be imposed after calculating the Poisson brackets. So for an arbitrary constraint, $\chi(q, p)$, primary or secondary:

$$
\left\{\chi_{i}, H_{T}\right\}_{P . B .} \approx 0
$$


There is a more important classification of constraints:

A constraint is called first class, if its Poisson bracket with all other constraints vanishes, a well-known example of which is the electromagnetic field [23]. First class constraints arise from symmetries of the action which in the electromagnetic case is the gauge invariance. The second class constraints are those with a non-zero and non-singular Poisson brackets,

$$
\left\{\chi_{M}, \chi_{N}\right\}_{P . B .} \equiv C_{M N}
$$

with $\operatorname{det} C \neq 0$.

According to Dirac, to quantize a system with second class constraints, the commutators of any two functions on the phase space should be given in terms of a modified bracket, Dirac bracket,

$$
[A, B]=i\{A, B\}_{D \cdot B .}
$$

where

$$
\{A, B\}_{D . B .}=\{A, B\}_{P . B .}-\left\{A, \chi_{M}\right\}_{P . B .}\left(C^{-1}\right)^{M N}\left\{\chi_{N}, B\right\}_{P . B .} .
$$

One can easily verify that the Dirac bracket satisfies the algebraic relations of the Poisson bracket. The important property of the Dirac bracket is that for an arbitrary $A$ and for all constraints $\chi_{M}$

$$
\{\chi, A\}_{D . B .}=0 .
$$

So, using the Dirac brackets instead of Poisson bracket, the weak equations may be written as strong equalities.

For second class constraints we can always find a canonical transformation which maps the set of the constrained variables, $q_{\alpha}, p_{\alpha}, \alpha=1, \ldots, N$, and the constraints $\chi_{M}, M=1, \ldots, n$, to a set $Q_{i}, P_{i} ; \xi_{M}, i=1, . ., N-n$, such that $\xi_{M}=0$ identically, and $Q, P$ are unconstrained [24], i.e.

$$
\{A, B\}_{D . B .}=\{A, B\}_{P . B . \text { in terms of } Q, P}=\frac{\partial A}{\partial Q_{i}} \frac{\partial B}{\partial P_{i}}-\frac{\partial B}{\partial Q_{i}} \frac{\partial A}{\partial P_{i}} .
$$

The set of $(Q, P)$ span the reduced phase space, and it has been shown that the quantization on this reduced phase space with the canonical commutators is equivalent to the Dirac quantization on the constrained phase space. 


\section{Boundary Conditions as Dirac Constraints}

In this section, having equipped ourselves with the Dirac bracket, we return to the problem of quantization of usual open strings which in the simplest case is governed by

$$
S=\frac{1}{4 \pi \alpha^{\prime}} \int\left(\left(\partial_{\tau} X^{i}\right)^{2}-\left(\partial_{\sigma} X^{i}\right)^{2}\right) d \sigma d \tau
$$

Variation of the above action reduces to the equations of motion: $\partial_{\tau}^{2} X^{i}=\partial_{\sigma}^{2} X^{i}$ and the Neumann boundary conditions, $\left.\partial_{\sigma} X^{i}\right|_{0, \pi}=0$. Treating the boundary conditions as the Dirac constraints, we try to quantize the theory directly by means of the Dirac method, without recourse to the mode expansions. Our point of view here, treating the boundary conditions as constraints, is justified and discussed in more detail in [25].

The total Hamiltonian of the open strings is

$$
H=\frac{1}{4 \pi \alpha^{\prime}} \int\left(\left(\partial_{\tau} X^{i}\right)^{2}+\left(\partial_{\sigma} X^{i}\right)^{2}\right) d \sigma d \tau+\left.u_{i} \partial_{\sigma} X^{i}\right|_{0 \text { or } \pi}
$$

with the canonical momenta

$$
2 \pi \alpha^{\prime} P^{i}(\sigma, \tau)=\partial_{\tau} X^{i}
$$

and the primary constraints: $\left.\Phi_{i} \equiv \partial_{\sigma} X^{i}\right|_{0}=0$. The boundary conditions at the other end of the string $(\sigma=\pi)$ can be worked out similarly.

Now, the consistency of the constraints,

$$
\left\{\Phi_{i}, H\right\}_{P . B .}=\left.2 \pi \alpha^{\prime} \partial_{\sigma} P^{i}\right|_{0}
$$

gives a new set of constraints; and therefore it is of the $i i)$ kind. Calling them $\Psi_{i}$, we can go on

$$
\left\{\Psi_{i}, H\right\}_{P . B .} \approx 0
$$

and find an equation for $u_{i}$. As discussed in [25], although $u_{i}$ is determined the constraint chain is not terminated, but along the arguments of [25] taking only $\left(\Phi_{i}, \Psi_{i}\right)$ from the constraint chain will give the proper result if at the end, we reconsider a normalization factor for $X_{i}$ and $P_{j}$ so that, their Dirac brackets (or commutators) become $\delta_{i j}$. Since the Poisson brackets of the constraints are not zero, we are dealing with second class constraints,

$$
\begin{gathered}
\left\{\Phi_{i}, \Phi_{j}\right\}_{P . B .}=0 \quad, \quad\left\{\Psi_{i}, \Psi_{j}\right\}_{P . B .}=0 \\
\left\{\Phi_{i}, \Psi_{j}\right\}_{P . B .}=\left\{\left.\partial_{\sigma} X^{i}\right|_{0},\left.\partial_{\sigma} P^{j}\right|_{0}\right\}_{P . B .}=\int_{0}^{\pi}\left\{\partial_{\sigma} X^{i}(\sigma), \partial_{\sigma} P^{i}\left(\sigma^{\prime}\right)\right\}_{P . B .} \delta(\sigma) \delta\left(\sigma^{\prime}\right) d \sigma d \sigma^{\prime} .
\end{gathered}
$$


To overcome the difficulty of dealing with the delta function derivatives at the boundaries, we discretize $\sigma$, denoting the steps by $\epsilon$, and take the $\epsilon \rightarrow 0$ at the end; thus

$$
\Phi^{i}=\frac{1}{\epsilon}\left(X_{1}^{i}-X_{0}^{i}\right)
$$

With the discrete Lagrangian conjugate momenta:

$$
2 \pi \alpha^{\prime} P_{0}^{i}=\epsilon \dot{X}_{0}^{i} \quad, \quad 2 \pi \alpha^{\prime} P_{1}^{i}=\epsilon \dot{X}_{1}^{i} .
$$

The $X_{n}^{i}, P_{n}^{i}, n=0,1$ have the usual Poisson brackets

$$
\begin{gathered}
\left\{X_{n}^{i}, X_{m}^{j}\right\}=0, \\
\left\{P_{n}^{i}, P_{m}^{j}\right\}=0 \\
\left\{X_{n}^{i}, P_{m}^{j}\right\}=\delta^{i j} \delta_{m n} .
\end{gathered}
$$

The secondary constraints in the discretized version are

$$
\Psi^{i}=P_{1}^{i}-P_{0}^{i}
$$

hence

$$
\left\{\Phi_{i}, \Psi_{j}\right\}_{P . B .}=\frac{1}{\epsilon}\left\{X_{1}^{i}-X_{0}^{i}, P_{1}^{j}-P_{0}^{j}\right\}=\frac{2}{\epsilon} \delta^{i j} .
$$

It should be emphasized that, we use the discrete language only as a method for calculating the commutators and at the end the objects surviving in the $\epsilon \rightarrow 0$ limit are physical. The $C$ matrix takes the form

$$
\left(\begin{array}{cc}
0 & \frac{2}{\epsilon} \delta^{i j} \\
\frac{-2}{\epsilon} \delta^{i j} & 0
\end{array}\right),
$$

and is invertible. For simplicity and to visualize how the Dirac method goes through, let us take the boundary conditions only on one of $X^{i}$ 's. In this case $C$ is a $2 \times 2$ matrix and

$$
C^{-1}=\frac{\epsilon}{2}\left(\begin{array}{cc}
0 & -1 \\
1 & 0
\end{array}\right)
$$

To quantize the theory we calculate the Dirac brackets and find

$$
\left\{X^{i}(\sigma), X^{j}\left(\sigma^{\prime}\right)\right\}_{D . B .}=0-\left\{X^{i}(\sigma), \chi_{M}\right\}_{P . B .}\left(C^{-1}\right)^{M N}\left\{\chi_{N}, X^{i}(\sigma)\right\}_{P . B .}=0
$$


The Dirac bracket for two $P^{i}(\sigma)$ 's similarly vanishes, but $\left\{X^{i}(\sigma), P^{j}\left(\sigma^{\prime}\right)\right\}_{D . B}$. will have a slightly different form near the boundary, i.e. at $\sigma, \sigma^{\prime}=0, \epsilon$ which can be removed by normalizing $X$ and $P$ by a factor of $\sqrt{2}$. The above Dirac brackets can be understood in terms of Maskawa-Nakajima theorem, [24] too. We can easily find the reduced phase space and the unconstrained variables, which are

$$
X^{i}=\frac{X_{1}^{i}+X_{0}^{i}}{2} \quad, \quad P^{i}=P_{1}^{i}+P_{0}^{i}
$$

and the identically vanishing constraints:

$$
X_{1}^{i}-X_{0}^{i} \quad, \quad P_{1}^{i}-P_{0}^{i}
$$

Comparing the method we used here with the usual open strings quantization based on the mode expansion, we find that in the mode expansion method, to impose the boundary conditions, we put coefficients of the Sine terms in the expansion equal to zero. This is equivalent to considering the secondary constraints and also going to the reduced phase space, and performing the quantization on these unconstrained set of variables. Hence in the Neumann boundary conditions, the mode expansion quantization and the Dirac method give the same quantum theory.

One can similarly apply the Dirac bracket method to the strings with Dirichlet boundary conditions. Again there are secondary constraints and the $C$ matrix is invertible, giving $\left\{X^{i}(\sigma), X^{j}\left(\sigma^{\prime}\right)\right\}_{D . B .}=0,\left\{P^{i}(\sigma), P^{j}\left(\sigma^{\prime}\right)\right\}_{D . B .}=0$. The reduced phase space is the one on which the $X_{0}, P_{0}$ are both omitted, which is again the same as what we have in the mode expansions; there we only choose the Sine, which is equivalent to working in the reduced phase space.

\section{Dirac Quantization For Mixed Boundary Conditions}

In this section we consider a more general case, the open strings with mixed boundary conditions. Let us take the open strings ending on a p-brane which is spanned by $X^{0}, X^{1}, \ldots, X^{p}$, assuming that we have turned on a constant $B_{\mu \nu}(\mu, \nu=0, \ldots, p)$ background and also we have a constant $F_{\mu \nu}$ ending on the brane. As explained earlier since the background $B$ is constant, it does not appear in the equation of motion and only appears in the boundary 
conditions

$$
\begin{aligned}
\partial_{\tau}^{2} X^{\nu} & =\partial_{\sigma}^{2} X^{\nu} \\
\partial_{\sigma} X^{\mu}+\mathcal{F}_{\mu \nu} \partial_{\tau} X^{\nu} & =0 \text { at } \sigma=0, \pi .
\end{aligned}
$$

The above equations enjoy two $U(1)$ symmetries explained earlier and hence the solution of them also have these symmetries. Calculating the conjugate momenta of $X^{\mu}$

$$
2 \pi \alpha^{\prime} P^{\mu}(\sigma, \tau)=\partial_{\tau} X^{\mu}+B_{\nu}^{\mu} \partial_{\sigma} X^{\nu}+A^{\mu}(\delta(\sigma-\pi)-\delta(\sigma)),
$$

we see that the momentum is not gauge invariant under either of these $U(1)$ 's. The $U(1)$ acting only on $A$, is similar to the familiar electrodynamics gauge symmetry and at the end survives in our quantized theory. Hence by choosing a gauge, we fix the $U(1)$ which only acts on $A^{\mu}$ field (1.8). For constant $F_{\mu \nu}, \lambda$ can be chosen so that

$$
A_{\mu}=\frac{-1}{2} F_{\mu \nu} X^{\nu}
$$

To quantize the theory, we consider the mixed boundary conditions as constraints and quantize the system by the Dirac method. Running the Dirac machinery first we should build the Hamiltonian from the action:

$$
H=\frac{1}{4 \pi \alpha^{\prime}} \int\left(\left(\partial_{\tau} X^{\mu}\right)^{2}+\left(\partial_{\sigma} X^{\mu}\right)^{2}\right) d \sigma .
$$

$\partial_{\tau} X^{\mu}$ are related to momenta through (4.2). We will put $2 \pi \alpha^{\prime}$ equal to one and reintroduce in our results at the end. Without loss of generality, we assume the electric mixing, i.e. $\mathcal{F}_{0 \nu}, B_{0 \nu}$ to be zero. The boundary conditions, or in other words, the primary constraints are

$$
\Phi^{i}=\partial_{\sigma} X^{i}+\left.\mathcal{F}_{i j} \partial_{\tau} X^{j}\right|_{\sigma=0} \quad \text { or } \pi=0 . \quad i, j=1,2, \ldots, p .
$$

Here the calculations are presented explicitly only for $\sigma=0$; for the other end $(\sigma=\pi)$, which is very similar to $\sigma=0$, we only give the results.

According to Dirac prescription, we should verify if there are any other constraints:

$$
\left\{\Phi_{i}, H_{T}\right\}_{P . B .}=\int_{0}^{\pi}\left\{\partial_{\sigma} X^{i}(\sigma)+\mathcal{F}_{i j} \partial_{\tau} X^{j}(\sigma), H\right\} \delta(\sigma) d \sigma+u_{j}\left\{\Phi_{i}, \Phi_{j}\right\}_{P . B .} .
$$

The $\left\{\Phi_{i}, \Phi_{j}\right\}_{P . B}$. in the above is ill-defined; hence the two terms should vanish independently; which leads to secondary constraints [25].

To avoid the ambiguities due to $\delta(\sigma)$ derivatives, we use the discretized form of the strings:

$$
\Phi^{i}=X_{1}^{i}-X_{0}^{i}+\epsilon \mathcal{F}_{i j} \partial_{\tau} X_{0}^{j},
$$


and the related part of the Hamiltonian

$$
H=\frac{\epsilon}{2}\left(\dot{X}_{0}^{2}+\dot{X}_{1}^{2}\right)+\frac{1}{2 \epsilon}\left[\left(X_{1}-X_{0}\right)^{2}+\left(X_{2}-X_{1}\right)^{2}\right]
$$

with

$$
\begin{gathered}
P_{0}^{i}=\epsilon \dot{X}_{0}^{i}+B_{i j}\left(X_{1}-X_{0}\right)_{j}+\frac{1}{2} F_{i j} X_{0 j} \\
P_{1}^{i}=\epsilon \dot{X}_{1}^{i}+B_{i j}\left(X_{2}-X_{1}\right)_{j} .
\end{gathered}
$$

$P_{n}^{i}, X_{n}^{i}, n=0,1$ have the usual canonical Poisson brackets (3.7).

The secondary constraints are

$$
\Psi^{i}=\left\{\Phi_{i}, H\right\}=\frac{1}{\epsilon}(\mathbf{1}-\mathcal{F} B)^{i j}\left(P_{1}^{j}-P_{0}^{j}+\frac{1}{2} F_{j k} X_{0 k}\right)=0
$$

which are not invariant under $\Lambda$-gauge transformation (1.9) explicitly. We must also check if there is any more constraints; this leads to an infinite chain of constraints, but as discussed in [25] taking all these constraints into account is equivalent to take only $\left(\Phi_{i}, \Psi_{i}\right)$ and normalize $X$ and $P$ with the same numeric factor so that $\left[X_{n}^{i}, P_{m}^{j}\right]=1 \delta_{m n} \delta^{i j}$.

At this stage following Dirac, the class of the constraints should be determined;

$$
\begin{gathered}
A_{i j} \equiv\left\{\Phi_{i}, \Phi_{j}\right\}_{P . B .}=2 \mathcal{F}^{i k} \mathcal{M}^{k j}-\mathcal{F}^{i k} F^{k l} \mathcal{F}^{l j}, \\
D_{i j} \equiv\left\{\Phi_{i}, \Psi_{j}\right\}_{P . B .}=2 \mathcal{M}^{i j}-\mathcal{F}^{i k} F^{k j} \\
\left\{\Psi_{i}, \Psi_{j}\right\}=-F_{i j}
\end{gathered}
$$

with

$$
\mathcal{M}^{i j}=\left(\mathbf{1}-\mathcal{F}^{2}\right)^{i j}
$$

Since the Poisson brackets of constraints are non-zero we may be dealing with second class constraints; to check that and to quantize the system we should find the $C$ matrix, the Poisson brackets of the constraints. In the general p-brane case $C$ is a $2 p \times 2 p$ matrix composed of $A, D, F$ matrices defined in (4.11):

$$
\begin{gathered}
C=\left(\begin{array}{cc}
A & D \\
-D^{t} & -F
\end{array}\right) . \\
\operatorname{det} C=\operatorname{det}\left(-A F+D D^{t}\right) .
\end{gathered}
$$

The above determinant, like the $C$ itself, is $\Lambda$-gauge dependent, but in any gauge, for real $\Lambda,(4.14)$ is non-zero and hence, $C$ is invertible. Therefore the constraints are in fact second 
class. To visualize how the Dirac method works, without loss of generality, we confine ourselves to the $D_{2}$-brane, i.e. $p=2$, in which $C$ is a $4 \times 4$ matrix. We are now ready to calculate the basic Dirac brackets:

$$
\begin{gathered}
\left\{X_{0}^{i}, X_{0}^{j}\right\}_{\text {D.B. }}=\left((-2 \mathcal{M}+\mathcal{F} F)^{-1} \mathcal{F}\right)^{i j}, \\
\left\{X_{0}^{i}, X_{1}^{j}\right\}_{D . B .}=0, \\
\left\{X_{1}^{i}, X_{1}^{j}\right\}_{\text {D.B. }}=\left((2 \mathcal{M}-2 \mathcal{F} F)^{-1} \mathcal{F}\right)^{i j} .
\end{gathered}
$$

Dirac brackets of $X^{i}$ for any point in the middle of the string are zero.

The important and new result we obtain here is that the Dirac brackets are not gauge invariant, and each of (4.15) or (4.16) could be set to zero by a gauge transformation. But, there is no gauge in which both of these brackets vanish.

Similarly we can find the basic Dirac brackets containing the momenta:

$$
\begin{gathered}
\left\{P_{0}^{i}, P_{0}^{j}\right\}_{D . B .}=\frac{1}{4} F^{i j}, \\
\left\{P_{0}^{i}, P_{1}^{j}\right\}_{D . B .}=0, \\
\left.\left\{P_{1}^{i}, P_{1}^{j}\right\}_{D . B .}=\frac{-1}{2}((\mathcal{M}-\mathcal{F} F) F(2 \mathcal{M}-F \mathcal{F}))^{-1}\right)^{i j} .
\end{gathered}
$$

and

$$
\begin{gathered}
\left\{X_{0}^{i}, P_{0}^{j}\right\}_{\text {D.B. }}=\frac{1}{2} \delta^{i j} \quad, \quad\left\{X_{0}^{i}, P_{1}^{j}\right\}_{\text {D.B. }}=\frac{1}{2} \\
\left\{X_{1}^{i}, P_{0}^{j}\right\}_{\text {D.B. }}=\frac{1}{2} \delta^{i j} \quad, \quad\left\{X_{1}^{i}, P_{1}^{j}\right\}_{\text {D.B. }}=\frac{1}{2} \delta^{i j} .
\end{gathered}
$$

To find the proper Dirac brackets, we should normalize the $X_{n}^{i}$ and $P_{m}^{j}$ by a factor of $\sqrt{2}$ so that the brackets of (4.19) are in their usual form. Hence the brackets of (4.17) and (4.18) should be multiplied by a factor of 2 .

The quantum theory is obtained by replacing the Dirac brackets with $-\mathrm{i} \times$ commutators. Reintroducing the $2 \pi \alpha^{\prime}$ factor we have

$$
\begin{gathered}
{\left[X_{0}^{i}, X_{0}^{j}\right]=-2 \pi \alpha^{\prime} i\left(\mathcal{M}-\frac{1}{2} \mathcal{F} F\right)^{-1} \mathcal{F}} \\
{\left[X_{0}^{i}, X_{1}^{j}\right]=0} \\
{\left[X_{1}^{i}, X_{1}^{j}\right]=2 \pi \alpha^{\prime} i(\mathcal{M}-\mathcal{F} F)^{-1} \mathcal{F} .}
\end{gathered}
$$


In other words the open strings coordinates on the brane, along which $B_{i j}$ and $F_{i j}$ have a non-zero value, become noncommuting, and the noncommutativity being gauge dependent, but not removable.

A special gauge which has been discussed earlier in $[8,9]$ is:

$$
F=0 \quad \text { or } \quad B=\mathcal{F} \text {. }
$$

In this gauge commutators take a simpler form

$$
\begin{gathered}
{\left[X_{0}^{i}, X_{0}^{j}\right]=-\left[X_{1}^{i}, X_{1}^{j}\right]=-2 \pi \alpha^{\prime} i\left(\mathcal{F} \mathcal{M}^{-1}\right)^{i j},} \\
{\left[X_{0}^{i}, X_{1}^{j}\right]=0}
\end{gathered}
$$

and

$$
\begin{gathered}
{\left[X_{n}^{i}, P_{m}^{j}\right]=\delta^{i j}\left(\delta_{n 0}+\delta_{n 1}\right)\left(\delta_{m 0}+\delta_{m 1}\right),} \\
{\left[P_{n}^{i}, P_{m}^{j}\right]=0 .}
\end{gathered}
$$

In the last part of this section we will compare our results in this gauge $(F=0)$ with the previous works, based on the mode expansions.

Boundary Conditions at $\sigma=\pi$

To discuss the noncommutativity of open strings center of mass we should also consider the boundary conditions at the other end of the open string. There are some choices: The boundary conditions can be Neumann, Dirichlet or mixed with the same or different mixing parameter, $\mathcal{F}_{i j}^{\prime}$. The open strings with Mixed boundary conditions at one end $(\sigma=0)$, and Neumann (Dirichlet) at the other end $(\sigma=\pi)$ are reffered to as $M N(M D)$ strings, and the open strings with mixed boundary conditions at both ends but with different (or the same) mixing parameters, $M M^{\prime}(M M)$. The first two cases, $M N$ and $M D$, happen when a mixed p-brane is parallel to a $D_{p}$-brane and $D_{p-2}$-brane respectively [5]. $M M$ strings appear when the open string has both ends on the same mixed brane.

To study the most general case we consider the $M M^{\prime}$ strings which recovers the $M M$, $M D$ and $M N$ as special cases. Since the calculations are very similar to what we have done earlier, we will only quote the results here

$$
\begin{gathered}
\left\{X_{\pi}^{i}, X_{\pi}^{j}\right\}_{\text {D.B. }}=\left(\left(2 \mathcal{M}^{\prime}-\mathcal{F}^{\prime} F^{\prime}\right)^{-1} \mathcal{F}^{\prime}\right)^{i j}, \\
\left\{X_{\pi}^{i}, X_{\pi-\epsilon}^{j}\right\}_{D . B .}=0 \\
\left\{X_{\pi-\epsilon}^{i}, X_{\pi-\epsilon}^{j}\right\}_{D . B .}=\left(\left(-2 \mathcal{M}^{\prime}+2 \mathcal{F}^{\prime} F^{\prime}\right)^{-1} \mathcal{F}^{\prime}\right)^{i j},
\end{gathered}
$$


Equations (4.23-25) compared to similar results at $\sigma=0,(4.15-17)$, have an extra minus sign. This can be understood intuitively noting that the open strings attached to branes are oriented open strings.

Noncommutativity of Open string Center of Mass

For $M M^{\prime}$ strings discussed above the commutator of the center of mass coordinates,

$$
X_{c . m .}^{i}=\int_{0}^{\pi} d \sigma \quad X^{i}(\sigma, \tau)=\epsilon \sum_{m=0}^{N} X_{m}^{i}
$$

is found to be

$$
\left[X_{c . m .}^{i}, X_{c . m .}^{j}\right]=\epsilon^{2} \sum_{m, n=0}^{N}\left[X_{n}^{i}, X_{m}^{j}\right]=4 \pi \alpha^{\prime} i\left\{\frac{1}{\sqrt{\operatorname{det} C}}(\mathcal{F} F \mathcal{F})^{i j}-\frac{1}{\sqrt{\operatorname{det} C^{\prime}}}\left(\mathcal{F}^{\prime} F^{\prime} \mathcal{F}^{\prime}\right)^{i j}\right\}
$$

where $\operatorname{det} C^{\prime}$ has the same form of $\operatorname{det} C(4.14)$, with $\mathcal{F}$ replaced by $\mathcal{F}^{\prime}$. The above relation is in general $\Lambda$-gauge dependent. But, in the important special case when the open string has both ends on the same mixed brane ( $M M$ string), the noncommutativity at the ends of these oriented open strings cancel each other and the center of mass commutators therefore vanishes and is not gauge dependent. In the other special cases, $M N$ and $M D$ open strings, we can choose a gauge $(F=0)$ in which the center of mass coordinates are commuting [5].

The noncommutativity of $M M$ open string components was first observed in [7], and in a more detailed work discussed in [8], and later in [9]. In the introduction we noted that the quantization methods used in [8] and [9], both based on the string mode expansions, and with the choice (1.22) are exactly equal. In this part we compare the results of the Dirac quantization method, with the previous works.

In order to make this comparison the Dirac method results should be rewritten in the special $\Lambda$-gauge, $F=0$, which leads to $(4.21)$, $(4,22)$ and also be translated into the continuum language:

$$
\begin{array}{cc}
X_{0}^{i}=\left.X^{i}(\sigma, \tau)\right|_{\sigma=0} \quad & X_{1}^{i}=X_{0}^{i}+\left.\epsilon \partial_{\sigma} X^{i}\right|_{\sigma=0} \\
P_{0}^{i}=\left.\epsilon P^{i}(\sigma, \tau)\right|_{\sigma=0} \quad & P_{1}^{i}=P_{0}^{i}+\left.\epsilon^{2} \partial_{\sigma} P^{i}\right|_{\sigma=0},
\end{array}
$$

and the similar relations at $\sigma=\pi$. Inserting these into $(4.21,23)$ we obtain

$$
\begin{gathered}
{\left[\left.X^{i}(\sigma, \tau)\right|_{\sigma=0},\left.X^{j}(\sigma, \tau)\right|_{\sigma=0}\right]=-\left[\left.X^{i}(\sigma, \tau)\right|_{\sigma=\pi},\left.X^{j}(\sigma, \tau)\right|_{\sigma=\pi}\right]=-2 i \pi \alpha^{\prime}\left(\mathcal{F} \mathcal{M}^{-1}\right)^{i j},} \\
{\left[\left.\partial_{\sigma} X^{i}\right|_{\sigma=0},\left.\partial_{\sigma} X^{j}\right|_{\sigma=0}\right]=0}
\end{gathered}
$$


and for the $X, P$ commutators,

$$
\begin{aligned}
& {\left[\left.X^{i}\right|_{\sigma=0},\left.P^{j}\right|_{\sigma=0}\right]=\frac{1}{\epsilon} \delta^{i j},} \\
& {\left[\left.\partial_{\sigma} X^{i}\right|_{\sigma=0},\left.P^{j}\right|_{\sigma=0}\right]=0,} \\
& {\left[\left.\partial_{\sigma} X^{i}\right|_{\sigma=0},\left.\partial_{\sigma} P^{j}\right|_{\sigma=0}\right]=0,}
\end{aligned}
$$

and also

$$
\left[\left.P^{i}\right|_{\sigma=0},\left.P^{j}\right|_{\sigma^{\prime}=0}\right]=0
$$

$\left[\left.X^{i}\right|_{\sigma=0},\left.\partial_{\sigma} X^{j}\right|_{\sigma=0}\right]$ in the $\epsilon \rightarrow 0$ (continuum limit) is not finite, and tends to infinity as $\frac{1}{\epsilon}$.

Comparing the Dirac's method results in the $\Lambda$-gauge in which $F$ is zero, (4.28), with (1.23) one finds that they are in exact agreement.

The other commutators, i.e. $\left[\partial_{\sigma} X, \partial_{\sigma} X\right],\left[\partial_{\sigma} X, P\right],\left[\partial_{\sigma} X, \partial_{\sigma} P\right],[P, P]$, can be worked out in the mode expansion method. Doing so, which is a straightforward calculation, we find the same results in both methods, the Dirac's and the mode expansion. One can go further and find all the commutators of mode expansion quantization from the Dirac method by considering the general $\sigma$, not only 0 and $\pi$, which we will not do it here.

Thus we see that the quantization methods based on mode expansion, [8] and [9], which rely on certain ad-hoc assumptions agree with the more fundamental one, the Dirac quantization procedure.

\section{Conclusions and Discussions}

We have tackled the problem of the noncommutativity of the internal coordinates of brane starting from the basic principles of Dirac quantization. We found that in spite of a large class of gauge symmetries, and gauge dependence of the form of the noncommutativity of the open strings attached to the brane in the presence of constant background antisymmetric field, there is no gauge in which the noncommutativity can be removed, i.e., noncommutativity of the coordinates of the open string-brane system is intrinsic. As the gauge changes, the details of the noncommutativity is altered, but it never goes away altogether. The position of noncommutativity is always on the brane. We discussed that, choosing the mixed boundary condition at one end, there are various choices for the boundary condition at the other end of open strings; it can be Neumann, Dirichlet, and Mixed with the same or different mixing parameters, referred to as $M N, M D, M M$ and $M M^{\prime}$ respectively. And showed that except 
for the $M M$ case, the center of mass coordinates of these open strings are noncommuting but $\Lambda$-gauge dependent, so that the center of mass noncommutativity can be removed in a certain gauge.

The $M M$ case physically describes the open strings having both ends on a mixed brane. For this case we showed that, although the open string end points are not commuting and this noncommutativity is $\Lambda$-gauge dependent, the center of mass coordinates are commuting which is a gauge independent result.

Having obtained the Connes' noncommutative structure as an inherent property of certain branes, many open questions can be addressed. The noncommutativity arguments based on mixed open strings quantization hold in both compact and non-compact branes. In the compact case [8], we showed that the $C^{*}$-algebra of the functions on noncommutative torus can be constructed through the open string zero modes. However, in the non-compact case, in spite of having noncommutative coordinates, since these functions are not normalizable, there is no $C^{*}$-algebra structure. In this case the brane is a Moyal plane. It is shown $[14,15]$ that the internal low energy effective theory living in this Moyal plane is noncommutative super Yang-Mills theory described by Moyal brackets [10]. Renormalizability of this theory, which has been checked up to one loop, is a question to be answered.

The other problem to be addressed is the dynamics of branes in noncommutative backgrounds. For the special case, zero-branes on a noncommutative two-torus, corresponding dynamics has been proposed to be a light cone formulation of M-theory with a non-zero three form; a generalization of Matrix-model conjecture. But, there are some suggestions that the Born-Infeld corrections to the Yang-Mills are not suppressed in the large $N$ limit, unlike the commutative case. Hence to have a proper formulation of Matrix-theory, Born-Infeld action on the noncommutative background should be considered and all the Matrix-theory arguments should be repeated for the noncommutative Born-Infeld, some of which have been considered by Hofman-Verlinde.

Another interesting open problem is the anomalous $U(1)$ symmetry. $\mathcal{F}=B-d A$, being invariant under both $\lambda$ and $\Lambda$ gauge symmetries, is a good parameter to describe the system at the classical level, but according to our explicit calculations these gauge symmetries do not survive at the quantum level. This can be understood noting that at the quantum level, the $\lambda$ gauge symmetry is lifted to the deformed $\mathrm{U}(1)$, which is the isometry group of the Moyal plane [20]. It seems that the supersymmetry arguments can help to understand this anomaly. Resolving this problem may shed light on the problem of $U(N)$ Born-Infeld theory and also the noncommutative Yang-Mills or DBI action, which is expected to have 
$\Lambda$-symmetry.

\section{Acknowledgements}

M.M Sh-J. would like to thank A. Shirzad for many helpful comments and discussions.

\section{References}

[1] J. Polchinski, Phys. Rev. Lett. 75(1995) 4724.

[2] E. Witten, Nucl. Phys. B460 (1996) 335.

[3] A. Hashimoto and W. Taylor IV, "Fluctuation Spectra of Tilted and Intersecting Dbranes From the Born-Infeld Action", Nucl. Phys. B503 (1997) 193, hep-th/9703217.

S. Gukov, I.R. Kelbanov, A.M. Polyakov, "Dynamics of (n,1)-strings", Phys. Lett. B423 (1998)64.

J.X. Lu, S. Roy, "(m,n)-string-like $D_{p}$-brane Bound States, hep-th/9904112.

J.X. Lu, S. Roy, "((F,D1),D3)-Bound State and Its T-dual Daughters", hep-th/9905014.

K. Hashimoto, "Born-Infeld Dynamics in Uniform Electric Field", hep-th/9905162.

[4] H. Arfaei and M.M. Sheikh-Jabbari, Nucl. Phys. B526 (1998) 278, hep-th/9709054.

[5] M.M. Sheikh-Jabbari, "More on Mixed Boundary Conditions and D-brane Bound States", Phys. Lett. B425 (1998) 48, hep-th/9712199.

[6] M.R. Garousi, "Super-String Scattering From D-brane Bound States", JHEP 9812 (1998) 008.

[7] F. Ardalan, H. Arfaei, M.M. Sheikh-Jabbari, "Mixed Branes and Matrix Theory on Noncommutative Torus", Proceeding of PASCOS 98, hep-th/9803067.

F. Ardalan, "String Theory, Matrix Model, and Noncommutative Geometry", hepth/9903117.

[8] F. Ardalan, H. Arfaei, M.M. Sheikh-Jabbari, "Noncommutative Geometry From Strings and Branes", JHEP 02 (1999) 016. 
[9] C.-S. Chu, P.-M. Ho, "Noncommutative Open Strings and D-branes", hep-th/9812219.

[10] A. Connes, M.R. Douglas, A. Schwarz, "Noncommutative Geometry and Matrix Theory: Compactification on Tori", JHEP 02 (1998) 003, hep-th/9711162.

[11] A.Connes, Noncommutative Geometry, Academic Press, 1994.

M. A. Rieffel, "Non-Commutative Tori-A Case Study of Non-Commutative Differentiable Manifolds", Contemp. Math. 105, 191-211 (1990).

[12] P.-M. Ho, "Twisted Bundle on Quantum Torus and BPS States in Matrix Theory", Phys. Lett. B434 (1998) 41, hep-th/9803166.

[13] M.M. Sheikh-Jabbari, "Open Strings in a B-field Background as Electric Dipoles", hepth/9901080, Phys. Lett. B455 (1999) 129.

[14] M. R. Douglas, C. Hull, "D-branes and Noncommutative Torus", JHEP 02 (1998) 008, hep-th/9711165.

[15] M.M. Sheikh-Jabbari, "Super Yang-Mills theory on Noncommutative Torus From Open String Interactions", Phys. Lett. B450 (1999) 119.

[16] P.-M. Ho, Y.-S. Wu, "Noncommutative Gauge Theories in Matrix Theory", hepth/9801147.

M. Li, "Comments on Supersymmetric Yang-Mills Theory on a Noncommutative Torus", hep-th/9802052.

Y.-K. E. Cheung, M. Krogh, "Noncommutative Geometry From 0-Branes in a Background B Field", Nucl. Phys. B528 (1998) 185, hep-th/9803031.

T. Kawano and K. Okuyama, "Matrix Theory on Noncommutative Torus", hepth/9803044.

M. Berkooz: "Non-local Field Theories and the Noncommutative Torus", hepth/9802069.

N. Nekrasov, A. Schwarz: "Instantons on Noncommutative $R^{4}$ and $(2,0)$ Superconformal Six-Dimensional Theory", Commun. Math. Phys. (1998) 198, hep-th/9802068.

H. Garcia-Compean, "On the Deformation Quantization Description of Matrix Compactifications", Nucl. Phys. B541 (1999) 651, hep-th/9804188. 
B. Morariu, B. Zumino, "Super Yang-Mills on Noncommutative Torus", hepth/9807198.

C. Hofman, E. Verlinde, "U-Duality of Born-Infeld on Noncommutative Two-Torus", JHEP 9812 (1998) 010, hep-th/9810116.

V. Schomerus, "D-branes and Deformation Quantization", hep-th/9903205.

M. Anazawa, "D0-branes in a H-field Background and Noncommutative Geometry", hep-th/9905055.

K. Hashimoto, "Born-Infeld Dynamics in Uniform Electric Field", hep-th/9905162.

[17] P.A.M. Dirac, "Lecture Notes on Quantum Mechanics", Yeshiva University New York, 1964. Also see, P.A.M. Dirac, Proc. Roy. Soc. London, ser. A, 246, 326 (1950).

[18] T. Banks, W. Fischler, S.H. Shenker and L. Susskind, Phys. Rev.D55 (1997) 5112.

L. Susskind, "Another Conjecture about Matrix Theory", hep-th/9704080.

[19] O. Bigatti and L. Susskind, "Review of Matrix theory", hep-th/9712072.

T. Banks, "Matrix Theory", hep-th/9710231.

W. Taylor IV, hep-th/9801182 and references therein.

[20] R. Dijkgraaf, E. Verlinde and H. Verlinde, Nucl. Phys. B500 (1997) 43, hep-th/9703030.

[21] C.P. Martin, D. Sanchez-Ruiz, "The one-loop UV Divergent Structure of U(1) YangMills Theory on Noncommutative $R^{4}$, hep-th/9903077.

M.M. Sheikh-Jabbari, "Renormalizability of Super Yang-Mills Theories on Noncommutative Torus", JHEP 06(1999) 015 , hep-th/9903107.

[22] R. G. Leigh, Mod. Phys. Lett. A4 28 (1989) 2767.

[23] S. Weinberg, "The Quantum Theory of Fields", vol. 1, Cambridge University Press.

[24] T. Maskawa and H. Nakajima, "Singular Lagrangian and the Dirac-Faddeev Method", Prog. Theo. Phys, Vol. 56, (1976) 1295.

[25] M.M. Sheikh-Jabbari and A. Shirzad, "Boundary Conditions as Dirac Constraints", hep-th/9907055. 
[26] F. Ardalan, S. Kim, F. Mansouri, Int. J. Mod. Phys.A (1997) 1183.

F. Mansouri, Exact Local Supersymmetry, Absence of Superpartners, and Noncommutative Geometries, hep-th/9704187. 